\title{
Total Thyroidectomy and Adjuvant Radioiodine Treatment Independently Decrease Locoregional Recurrence Risk in Childhood and Adolescent Differentiated Thyroid Cancer
}

Daria Handkiewicz-Junak ${ }^{1}$, Jan Wloch ${ }^{2}$, Jozef Roskosz ${ }^{1}$, Jolanta Krajewska ${ }^{1}$, Aleksandra Kropinska ${ }^{1}$, Lech Pomorski ${ }^{3}$, Aleksandra Kukulska ${ }^{1}$, Andrzej Prokurat ${ }^{4}, Z_{\text {Zbigniew Wygoda }}{ }^{1}$, and Barbara Jarzab ${ }^{1}$

\begin{abstract}
${ }^{I}$ Department of Nuclear Medicine and Endocrine Oncology, Maria Sklodowska-Curie Memorial Cancer Center and Institute of Oncology, Gliwice Branch, Gliwice, Poland; ${ }^{2}$ Department of Surgical Oncology, Maria Sklodowska-Curie Memorial Cancer Center and Institute of Oncology, Gliwice Branch, Gliwice, Poland; ${ }^{3}$ Department of Endocrinological and General Surgery, Medical University of Lodz, Lodz, Poland; and ${ }^{4}$ Department of Pediatric Surgery, Rydygier Medical University, Bydgoszcz, Poland
\end{abstract}

We sought to assess whether extensive surgical treatment, postsurgical radioiodine therapy, or both decrease the risk of locoregional recurrence $(\mathrm{LR})$ after curative primary treatment in children and adolescents diagnosed with differentiated thyroid cancer (DTC) at age $\leq 18 \mathrm{y}$. Methods: To determine the incidence of and identify predictive factors for thyroid bed recurrence (TBR) or lymph node recurrence (NR), we performed a chart review and retrospective multivariate Cox regression analysis on 235 patients with DTC diagnosed at age $\leq 18 \mathrm{y}$ and managed with curative intent at our tertiary referral center from 1973 to 2002; 40 of these patients had distant metastases at diagnosis. We also determined overall and recurrence-free survival and generated curves for these variables using Kaplan-Meier and Cox univariate analysis. Results: During a median follow-up of $82 \mathrm{mo}$ (range, 5-402 mo), no DTC-related deaths occurred, 203 (86\%) children remained recurrence-free, and $32(14 \%)$ children had LR, including TBR in 9 ( $28 \%$ of LR), NR in 20 (63\% of LR), and both in 3 ( $9 \%$ of LR). Among patients treated with radical intent and showing no distant metastases, the most recent thyroglobulin level was $<1 \mathrm{ng} / \mathrm{mL}$ in all but $4 \%$ of cases. The median time from the first surgery to LR was $37 \mathrm{mo}$ (range, 9-280 mo). In multivariate analysis, significant risk factors for TBR were less than total thyroidectomy and lack of postsurgical radioiodine treatment (respective risk increases of $9.5[P=0.04]$ and 11 times $[P=0.03])$. For NR, classic papillary histology, incomplete primary lymph node management (i.e., lack of modified lymphadenectomy of affected lymph nodes or lack of confirmation of disease-free nodes by intraoperative staging), and absence of adjuvant radioiodine therapy were independent significant predictive factors that increased the recurrence risk by $1.9(P=$ $0.02)$, $3.3(P=0.02)$, and $3.2(P=0.02)$ times, respectively. Age or sex did not correlate with LR risk. Conclusion: In DTC patients $\leq 18$ y of age, extensive initial therapy - consisting of total thyroidectomy combined with modified lymphadenectomy

Received Aug. 21, 2006; revision accepted Feb. 22, 2007.

For correspondence or reprints contact: Barbara Jarzab, MD, PhD, Wybrzeże Armii Krajowej 15, 44-100 Gliwice, Poland.

E-mail: bjarzab@io.gliwice.pl

COPYRIGHT @ 2007 by the Society of Nuclear Medicine, Inc. performed in case of lymph node metastases and followed by radioiodine therapy - is associated with a substantial decrease of DTC LR risk.

Key Words: differentiated thyroid cancer; recurrence; children; therapy; radioiodine

J Nucl Med 2007; 48:879-888

DOI: 10.2967/jnumed.106.035535

A lthough experts (1-3) unequivocally agree that surgical resection is the most effective treatment for differentiated thyroid cancer (DTC) in children and adolescents, the choice of the therapeutic approach to pediatric DTC remains controversial. The disagreement relates to both the indication for total thyroidectomy and the extent of cervical lymph node dissection. Some investigators recommend routine near-total or total thyroidectomy $(2,4-8)$, arguing that such surgery removes all malignant thyroid tissue, improves patient outcome, and makes follow-up more reliable. Other investigators advocate less aggressive treatment to decrease the risk of surgical complications (9-11).

The controversy also extends to postoperative radioiodine therapy (12). This adjuvant modality has a long history in children, with well-documented benefits in the detection and treatment of lung metastases $(13,14)$. However, this intervention's effect on reducing locoregional recurrence (LR) in children is less clear, having been addressed in only a few studies (15). It is also unclear whether the use of both total thyroidectomy and postoperative radioiodine is complementary in young DTC patients. In the literature on adult patients, studies demonstrate the recurrence-reducing effect of postsurgical radioiodine (16); however, in lowerrisk patients, the efficacy of this modality remains unproven (17). Additionally, only one major study has shown an independent effect of radioiodine ablation on reduction of 
DTC-related mortality (2); this observation is not shared by other authors (11). Thus, no definitive recommendation is given on this intervention's routine use $(18,19)$.

The questions surrounding the best treatment for pediatric DTC cannot be resolved by randomized trials because of the small number of cases, the long natural history, and the relatively good prognosis of the disease (12). Analysis of retrospective data with multivariate evaluation of potential predictive factors for LR may be an alternative choice in tailoring optimal treatment algorithms. For this reason, we conducted the present study involving such analysis in a relatively large series of 235 children and adolescents with DTC who were treated, monitored, or both with curative intent at our tertiary referral cancer center over the $30-y$ period of 1973-2002.

\section{MATERIALS AND METHODS}

\section{Study Objectives and Outcomes}

We sought to determine the incidence and timing of-and predictive factors for- $\mathrm{LR}$ in the forms of thyroid bed recurrence (TBR) or lymph node recurrence (NR) in patients diagnosed with operable DTC at age $\leq 18$ y ("pediatric patients"). Specifically, we sought to assess whether lack of total thyroidectomy, of complete lymph node management, or of adjuvant radioiodine therapy (as defined below) independently contributed to the likelihood of LR.

We chose LR as the primary outcome measure for our study for 3 reasons. First, LR is a meaningful clinical event, as it requires at least one additional major therapeutic intervention and may heighten the risk of distant metastasis or DTC-related mortality. Second, our postprimary treatment follow-up protocol provided a high level of confidence that the neck had been rendered diseasefree by primary treatment and, hence, that any LR represented a true recurrence rather than previously undiagnosed residual disease. Third, no DTC-related death occurred in our patients within the observation period, so cause-specific mortality was unavailable as an outcome measure.

\section{Patients, Primary Treatment, and Follow-up}

The 235 patients in this series comprise all patients diagnosed with DTC at age $\leq 18$ y who were treated, monitored, or both with curative intent at our tertiary referral center between 1973 and 2002. To avoid the bias caused by inclusion of patients with known residual local disease, we excluded from the study 4 children who underwent surgery with palliative intent within this period. The study population represents approximately $3.6 \%$ of all patients referred to our center with the diagnosis of DTC during the years 1973-2002. The present series includes 109 patients who were statistically analyzed in a previous study on childhood and adolescent DTC (6).

Of the 235 children in the study, $199(85 \%)$ were diagnosed after 1990. The relatively high proportion of the study population treated after this date was due to the improved access to tertiary centers as well as the increased awareness and earlier diagnosis of DTC by pediatricians. Fifty-eight $(25 \%)$ of the series had their first thyroid surgery performed at our hospital, whereas the other $177(75 \%)$ underwent thyroid surgery at another center and then were referred to our hospital for completion surgery, radioiodine therapy, or both. Fifteen $(6 \%)$ children were referred only at the time of a diagnosis of LR.

Distant metastases were detected at the primary DTC diagnosis in $40(17 \%)$ of the 235 children. Primary treatment was considered to have rendered all of them free of local disease. In 3 (1.2\%) of the 235 patients, distant metastases were detected as the first and only manifestation of DTC recurrence; in another $4(1.7 \%)$ patients, distant metastases accompanied LR (Table 1). In 3 of the 47 patients with distant metastases, the lesions were not radioiodineavid and were detected on radiologic examinations.

Our follow-up protocol consisted of physical examination, diagnostic ${ }^{131}$ I whole-body scanning (dxWBS), and, after 1990, neck ultrasonography (US) and both suppressed and stimulated serum thyroglobulin ( $\mathrm{Tg}$ ) testing (with Tg recovery as the measure of interference), supplemented as necessary by plain radiography or, after 1992, CT. dxWBS was performed after 4 wk of thyroid hormone withdrawal (THW) or, in 4 recently treated patients, after recombinant human thyroid-stimulating hormone (TSH) administration.

This protocol was used to assess the completeness of the thyroid operation and the presence of residual disease 3 mo after surgery or, in patients who had surgery elsewhere, at their initial visit to our clinic. With the exception of dxWBS, the protocol was repeated every 6 mo for the $5 \mathrm{y}$ after the most recent therapy and yearly thereafter. dxWBS was performed 6-12 mo after each radioiodine therapy and, in cases of negative WBS and undetectable Tg, at 5-y intervals thereafter. In cases of increased serum Tg level, suspicious neck US findings, or both, diagnosis of LR was confirmed with fine-needle aspiration biopsy.

Within the year after the initial operation, 135 (57\%) children underwent further surgery because of insufficiently extensive surgery. To decrease the risk of permanent surgical complications, further operations due to incomplete surgery were scheduled for 2-3 mo after the most recent resection. Unless diagnosed with relapse, children referred $>1$ y after the initial operation were only monitored, without additional surgery or radioiodine treatment.

Since 1990, during thyroid surgery performed in our center, all children had bilateral surgical biopsy of any enlarged lymph node or, when no enlarged nodes were observed, of a random lymph node. If histopathology was positive, the surgery was extended to include modified lymphadenectomy. Before 1990, modified lymphadenectomy and, in a few cases, Crile's operation were performed only when lymph nodes were palpable. The extent of lymph node surgery performed elsewhere was assessed on the basis of the surgical and histopathology reports.

Therapeutic ${ }^{131}$ I was given after surgery to 174 (74\%) children and adolescents, $143(82 \%)$ of whom had received total thyroidectomy (Table 2). Children with total or near-total thyroidectomy

TABLE 1

Relationship Between LR and Distant Metastases

\begin{tabular}{cccc}
\hline LR & $\begin{array}{c}\text { Distant } \\
\text { metastases at } \\
\text { diagnosis }\end{array}$ & $\begin{array}{c}\text { Late distant } \\
\text { metastases }^{*}\end{array}$ & $\begin{array}{c}\text { No signs of } \\
\text { distant metastases } \\
\text { throughout study }\end{array}$ \\
\hline No & $37 / 203(18)$ & $3 / 203(1)$ & $163 / 203(80)$ \\
Yes & $3 / 32(9)$ & $4 / 32(13)$ & $25 / 32(78)$
\end{tabular}

${ }^{*}$ Diagnosed $>2$ y after primary treatment.

Values in parentheses are percentages. 
TABLE 2

Characteristics of 235 Children at End of Primary Treatment

\begin{tabular}{|c|c|c|}
\hline Characteristic & Variable & Number (\%) \\
\hline \multicolumn{3}{|c|}{ Patient-related factors } \\
\hline \multirow[t]{2}{*}{ Sex } & Female & $179 / 235(72)$ \\
\hline & Male & $66 / 235(28)$ \\
\hline \multirow{3}{*}{ Age $(y)$} & $<10$ & 24/235 (10) \\
\hline & $\geq 10$ and $<15$ & $97 / 235(41)$ \\
\hline & $15-18$ & $114 / 235(49)$ \\
\hline \multicolumn{3}{|c|}{ Tumor-related factors } \\
\hline \multirow[t]{3}{*}{ DTC histology } & Papillary, classic variant & 125/235 (53) \\
\hline & Papillary, follicular variant & $68 / 235(29)$ \\
\hline & Follicular & $42 / 235(18)$ \\
\hline \multirow[t]{6}{*}{ T stage, UICC 1997 classification* (data available in 141 cases) } & 1, unifocal & $1 / 141(<1)$ \\
\hline & 1, multifocal & 12/141 (9) \\
\hline & 2 & $97 / 141$ (69) \\
\hline & 3 & $13 / 141(13)$ \\
\hline & 4 & $18 / 141(8)$ \\
\hline & No data available & $94 / 235(40)$ \\
\hline \multirow[t]{3}{*}{ Lymph node metastases (lateral compartment) } & Yes & $106 / 235(45)$ \\
\hline & No & $66 / 235(28)$ \\
\hline & Not ruled out (no surgical biopsy) & $63 / 235(27)$ \\
\hline \multicolumn{3}{|c|}{ Treatment-related factors } \\
\hline \multirow[t]{4}{*}{ Treatment of thyroid gland } & Total Tx without ${ }^{131}$ I & $29(12)$ \\
\hline & Total Tx and ${ }^{131} \mathrm{I}$ & $143(61)$ \\
\hline & Less than total Tx without ${ }^{131}$ I & $32(14)$ \\
\hline & Less than total Tx with ${ }^{131}$ I & $31(13)$ \\
\hline \multirow[t]{12}{*}{ Lymph node management } & Complete $^{\dagger}$ and ${ }^{131}$ I & $129(55)$ \\
\hline & Lymphadenectomy & $69(29)$ \\
\hline & Negative surgical biopsy & $60(26)$ \\
\hline & Complete $^{\dagger}$ without ${ }^{131}$ I & $28(12)$ \\
\hline & Lymphadenectomy & $16(7)$ \\
\hline & Negative surgical biopsy & $12(5)$ \\
\hline & Incomplete ${ }^{\dagger}$ and ${ }^{131}$ | & $45(19)$ \\
\hline & Berry picking ${ }^{\dagger}$ & $15(6)$ \\
\hline & No surgery & $30(13)$ \\
\hline & Incomplete ${ }^{\dagger}$ without ${ }^{131}$ I & $33(14)$ \\
\hline & Berry picking ${ }^{\dagger}$ & $11(5)$ \\
\hline & No surgery & $22(9)$ \\
\hline \multicolumn{3}{|c|}{$\begin{array}{l}\text { *Information was unavailable on multifocality in } 82 \text { cases (35\%). } \\
\text { 'Detailed explanations of term are found in Material and Methods. } \\
\text { UICC = International Union Against Cancer; Tx = thyroidectomy. } \\
\text { "Less than total Tx" is thyroid operation that was not defined as total/near-total thyroidectomy and/or after which the postoperative } \\
\text { evaluation revealed total remnant volume }>2 \mathrm{~mL} \text { by sonography and/or thyroid bed uptake }>10 \% \text { "Berry picking" means selected } \\
\text { excision of metastatic lymph nodes; italic type indicates type of surgical procedures included in a given class of lymph node management. } \\
\text { Some percentage totals were affected by rounding to the nearest whole percentage. }\end{array}$} \\
\hline
\end{tabular}

and $<1 \%$ postoperative radioiodine uptake in the thyroid bed on $\mathrm{dxWBS}$ were not given radioiodine unless the stimulated Tg level exceeded $30 \mathrm{ng} / \mathrm{mL}$ postoperatively. The therapeutic ${ }^{131}$ I activity was adjusted to the child's age and disease stage. Children $<12-y-$ old were given $74.0-92.5 \mathrm{MBq} / \mathrm{kg}$ of body weight. Older children were given fixed activities: $2.2-3.7 \mathrm{GBq}$ as postsurgical adjuvant therapy when no distant metastases were known or $3.7 \mathrm{GBq}$ to treat known functional distant metastases. Patients received single activities of therapeutic radioiodine unless they were found to have functional distant metastases, in which case they received 2-6 courses of radioiodine with a mean cumulative activity of 11 GBq. Radioiodine was applied after at least 4 wk of THW, when serum TSH exceeded $25 \mathrm{mIU} / \mathrm{L}$. From 1993, postherapy WBS
(rxWBS), supplemented if needed by spot views of sites suggestive of distant metastases, was performed $72 \mathrm{~h}$ after therapeutic radioiodine application. Children were considered free of distant disease if there was no uptake on rxWBS and $\mathrm{Tg}$ was $<4 \mathrm{ng} / \mathrm{mL}$, when measured during the subsequent follow-up on $\mathrm{L}-3,5,3^{\prime}$, $5^{\prime}$-tetraiodothyronine (thyroxine; $\mathrm{T}_{4}$ ) therapy. This cutoff level was selected at $95 \%$ specificity, based on the institutional receiveroperating-characteristic curve (Fig. 1). Serum Tg concentration was monitored via an immunofluorometric assay with a functional sensitivity of $1 \mathrm{ng} / \mathrm{mL}$ (Wallac-Delfia).

After primary surgery, except during THWs for radioiodine treatment or scanning, children were given L-thyroxine to suppress TSH levels below $0.05-0.1 \mathrm{mIU} / \mathrm{mL}$. 
FIGURE 1. Institutional receiver-operating-characteristic curves for serum $\mathrm{Tg}$ measurements (based on $1,847 \mathrm{Tg}$ measurements on 1,122 patients of all ages with DTC) (20).

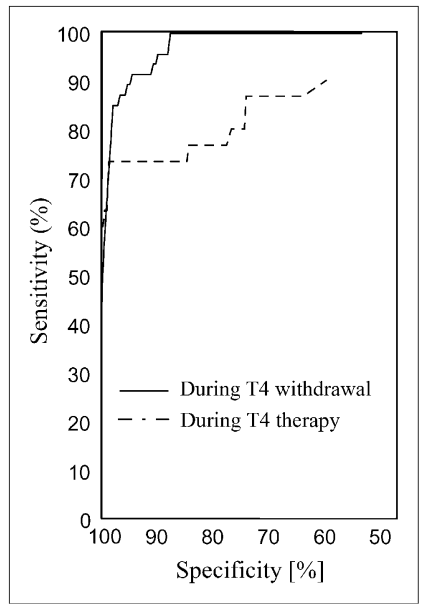

\section{Chart Review and Statistical Methods}

Charts were reviewed for LR, TBR, and NR and for the following potential predictive variables for these outcomes: sex, age at DTC diagnosis, tumor stage, tumor histology, extent of thyroid surgery, completeness of lymph node management, and presence of postoperative radioiodine therapy.

LRs were defined as any appearance of biopsy-confirmed DTC in the thyroid bed (TBR) or lymph nodes of the lateral compartment (NR) detected $>9$ mo after the initial operation and, in all cases, were positively verified by postoperative histopathologic examination. The indications for fine-needle biopsy were detection of any clinically suspicious lesion by physical examination or neck US, performed on routine evaluation or on detection of a rise in serum $\mathrm{Tg}$ concentration to $>4 \mathrm{ng} / \mathrm{mL}$. Any locoregional disease detected within 9 mo after the initial operation was regarded as grounds for completion primary surgery-and not as an LR.

We classified the completeness of thyroidectomy on the basis of both the surgical report and the latest examination performed within $1 \mathrm{y}$ of the initial surgery. Thyroidectomy was classified as "total or near-total" if (a) it was described as such in the surgical report and (b) the postoperative thyroid bed uptake on dxWBS or rxWBS was $<10 \%$ or, since 1990 , the total thyroid remnant volume on US was $<2 \mathrm{~mL}$. If at least one of these criteria was not met, the thyroidectomy was classified as "less than total." In all children with total or near-total thyroidectomy, postoperative stimulated TSH was $>25 \mathrm{mIU} / \mathrm{L}$, with the exception of 2 children in whom $23 \mathrm{mIU} / \mathrm{L}$ was accepted.

Lymph node management was classified as "complete" if modified lymph node dissection was performed in cases of lymph node involvement or if bilateral intraoperative surgical biopsy was negative. Lymph node management was considered "incomplete" in all other cases, including patients with selected excision of suspicious or metastatic lymph nodes ("berry-picking" surgery), a procedure performed only at the referring centers. The extent of lymph node operations performed outside our center was assessed on the basis of the surgical and histopathology reports.

LR-free survival was estimated with the Kaplan-Meier method as TBR-free survival (TBRFS) and NR-free survival (NRFS), defined as the time from the first operation until the first thyroid bed or lymph node event, respectively, or, if no event occurred, until the last follow-up visit to our center. Potential predictive variables were first evaluated in a Cox-Mantel univariate analysis and then by a multivariate regression Cox model in which all studied variables were included. A $P$ value $\leq 0.05$ was deemed significant. All statistical analyses were performed on the Statistica $\mathrm{Pl}$ for Windows package (Statsoft).

\section{RESULTS}

\section{Patient Characteristics and Observation Time}

Table 2 provides characteristics of the 235 children in our series. Slightly under $75 \%$ of our patients were female, roughly $50 \%$ were age $15-18 \mathrm{y}$, and $>75 \%$ had the papillary histotype. In general, most patients were intensively treated, with near-total or total thyroidectomy in $172(73 \%)$ patients, complete lymph node management in 157 (67\%) patients, and postoperative radioiodine in 174 (74\%) patients. The median observation time from DTC diagnosis to the last follow-up visit at our center was 82.2 mo (range, 4.6-402 mo). One hundred fifty-four $(65 \%)$ patients were observed for $\geq 5$ y and 60 (26\%) children were observed for $\geq 10 \mathrm{y}$. Seventy-four (31\%) children were lost to followup or moved to other centers. The subgroup lost to followup or moving to other centers had a median time similar to that of the last observation at our center $(81 \mathrm{mo}$; range, 4.6-231 mo) as did the subgroup not lost to follow-up.

\section{Outcomes}

During observation, LR was diagnosed in 32 (14\%) children. The median time from the first surgical intervention to the diagnosis of LR was 37 mo (range, 9-280 mo) and in 13 cases ( $41 \%$ of LR) the time was $>5$ y. In the Kaplan-Meier analysis, the disease-free survival was $90 \%$ after $5 \mathrm{y}, 84 \%$ after $10 \mathrm{y}$, and $82 \%$ after $15 \mathrm{y}$ (with 139, 49, and 12 patients, respectively, without recurrence under follow-up).

Nine (28\% of LR cases) children had TBR, 20 (63\% of LR cases) had NR, and 3 (9\% of LR cases) had both. In 4 patients (13\% of LR cases), LR was accompanied by detection of distant metastases. After a median 25 mo (range, 7-81 mo) following the diagnosis of the first LR, 7 children ( $22 \%$ of LR cases) were diagnosed with a second LR.

Serum $\mathrm{Tg}$ concentrations at the time of diagnosis of the first LR were available and evaluable in 23 (72\%) of the 32 patients with LR; in 2 additional children, Tg was considered inevaluable because of low $\mathrm{Tg}$ recovery. In the remaining 7 children, the analyte was not measured at the time of LR diagnosis because the LR had already been unequivocally demonstrated by fine-needle aspiration biopsy performed after findings were suspicious on physical examination, US, or both.

In $19(83 \%)$ of the 23 children with evaluable data, serum $\mathrm{Tg}$, measured during either $\mathrm{T}_{4}$ withdrawal or triiodothyronine $\left(\mathrm{T}_{3}\right)$ therapy, was elevated above the institutional cutoff at the time of LR diagnosis (Table 3). After treatment of recurrent disease, serum $\mathrm{Tg}$ decreased below our $1 \mathrm{ng} / \mathrm{mL}$ limit of detection in all but 1 child, whose $\mathrm{Tg}$ concentration was $1.7 \mathrm{ng} / \mathrm{mL}$. In addition, posttreatment $\mathrm{Tg}$ concentrations of the group with LR did not differ from those of children who never had disease recurrence or distant metastases. In the whole group of patients showing no 
TABLE 3

Serum Tg Levels in Children and Adolescents with DTC

\begin{tabular}{|c|c|c|c|c|c|}
\hline \multicolumn{2}{|c|}{ Time of Tg evaluation* } & Hormonal status & $\begin{array}{l}\text { Detectable } \mathrm{Tg}^{\dagger} \\
\quad(>1 \mathrm{ng} / \mathrm{mL})\end{array}$ & $\begin{array}{c}\text { Median } \\
\text { (range in } \mathrm{ng} / \mathrm{mL} \text { ) }\end{array}$ & $P$ \\
\hline \multirow{3}{*}{\multicolumn{2}{|c|}{$\begin{array}{l}\text { Before adjuvant }{ }^{131} \text { I therapy } \\
\text { At diagnosis of LR }\end{array}$}} & Off $\mathrm{T}_{4}$ therapy $(n=107)$ & $72(67)$ & $3.5(0.2-320)$ & \multirow{3}{*}{0.01} \\
\hline & & Off $\mathrm{T}_{4}$ therapy $(n=17)$ & $17(100)$ & $26.3(1.1-581)$ & \\
\hline & & On $T_{4}$ therapy $(n=6)$ & 2 (33) & $0.6(0.2-43)$ & \\
\hline \multirow[t]{2}{*}{$\begin{array}{l}\text { At last follow-up } \\
\text { (on } T_{4} \text { therapy) }\end{array}$} & $\begin{array}{l}\text { Children with recurrence } \\
\text { during follow-up }\end{array}$ & On $\mathrm{T}_{4}$ therapy $(n=30)$ & $1(3)$ & $0.4(0.2-1.7)$ & \multirow{2}{*}{ NS } \\
\hline & $\begin{array}{l}\text { Children without recurrence } \\
\text { during follow-up }\end{array}$ & On $\mathrm{T}_{4}$ therapy $(n=130)$ & $5(4)$ & $0.3(0.2-6.5)$ & \\
\hline
\end{tabular}

*Only cases with $\mathrm{Tg}$ recovery $>75 \%$ were included; children who had distant metastases were excluded.

${ }^{\dagger}$ Values in parentheses are percentages.

NS = not significant.

distant metastases, the most recent $\mathrm{Tg}$ level was $<1 \mathrm{ng} / \mathrm{mL}$ in all but $4 \%$ of cases.

Among the series as a whole, 1 death occurred, due to a second primary cancer (breast carcinoma) in a 33-y-old female, diagnosed with DTC at age $13 \mathrm{y}$ and treated with radioiodine $(2.2 \mathrm{GBq})$ during the fourth DTC recurrence, at age $25 \mathrm{y}$. Advanced breast cancer was diagnosed in this patient at age $31 \mathrm{y}, 6 \mathrm{y}$ after the single radioiodine administration.

\section{TBR}

The median time to TBR was $81 \mathrm{mo}$ (range, 36-280 mo). For the overall series, the rate of TBRFS was $99 \%$ at $5 \mathrm{y}$ $(n=149)$ and $94 \%$ at $10 \mathrm{y}(n=53)$ after initial thyroidectomy. Eleven (92\%) of 12 TBRs were diagnosed in patients treated with less than total thyroidectomy. Thus, the TBR rates were 1 of $172(<0.1 \%)$ for patients with near-total or total thyroidectomy and 11 of $63(17.4 \%)$ for patients with less than total thyroidectomy. No child with recurrence after less than total thyroidectomy had received ${ }^{131}$ I after primary surgery, but the only child with recurrence after total thyroidectomy had received postoperative radioiodine (Table 4).

In univariate analysis (data not shown), sex, age, and DTC histology did not correlate with TBRFS. However, both treatment-related factors-total thyroidectomy and radioiodine therapy—significantly decreased the risk of TBR $(P<0.001)$ (Figs. 2 and 3$)$. These effects were also significant in multivariate analysis (Table 4): Less than total thyroidectomy increased TBR risk by a factor of 9.5 (95\% confidence interval $[\mathrm{CI}], 1.2-78.1 ; P=0.04$ ) and lack of postoperative radioiodine independently increased the risk by a factor of $11.0(95 \% \mathrm{CI}, 1.3-94.7 ; P=0.03)$.

\section{NR}

The median time to NR was 17.0 mo (range, 9.1-279.9 mo). The NRFS was $92 \%$ at $5 \mathrm{y}(n=141)$ and $90 \%$ at $10 \mathrm{y}$ $(n=55)$ after initial thyroidectomy.

Eleven (48\%) of the 23 children with an eventual NR had been diagnosed with lymph node metastases to the lateral neck compartment at primary surgical treatment. These metastases were treated with modified lymphadenectomy in 4 and with excision of selected lymph nodes (berry-picking surgery) in 7 of the 11 patients. Three (13\%) of the 23 patients with an eventual NR had a negative surgical biopsy on primary treatment. No biopsy was obtained or no lymph node surgery was performed during primary treatment in 6 (26\%) of the 23 patients. Of the remaining 3 children, who had NR as well as TBR, 2 had selective excision of affected lymph nodes and the third child received no lymph node surgery as part of the primary treatment. Eight (35\%) of the 23 children with NR received radioiodine after primary surgery.

In univariate analysis, sex or age did not significantly influence the incidence of NRFS. As all NRs occurred in patients with the papillary histotype, a post hoc univariate analysis was performed to evaluate the difference between the classical versus the follicular variant of papillary DTC. This analysis found significantly more recurrences in children with the classical variant: The 10-y NRFS was $83 \%$ in children with this histologic subtype versus $95 \%$ in children with the follicular variant $(P<0.05)$.

Lymph node metastases or unknown lymph node status at thyroidectomy resulted in decreased NRFS when compared with biopsy-confirmed absence of lymph node involvement (Fig. 4). On the other hand, children with lymph node metastases at diagnosis but treated with modified neck lymphadenectomy had a NRFS similar to those with biopsynegative nodes (Fig. 5). Children who were treated with modified neck lymphadenectomy or who had negative surgical biopsy - that is, those classified as having "complete lymph node management" - had a 5-y NRFS of $96 \%(n=$ 157), compared with $81 \%$ in children who never underwent biopsy or surgery or who were treated only by excision of affected lymph nodes - that is, cases of "incomplete lymph node management" $(n=78)$. This difference was statistically significant $(P=0.02)$. Lack of radioiodine treatment after primary surgery was another significant risk factor for NR in univariate analysis $(P=0.00024$; Fig. 6$)$. The extent 
TABLE 4

Multivariate Analysis of Potential Predictive Factors for TBR or NR

\begin{tabular}{|c|c|c|c|c|}
\hline Factor (category or categories) & $\begin{array}{l}\text { No. of events/no. } \\
\text { of cases in category }\end{array}$ & $\begin{array}{l}\text { Odds ratio for } \\
\text { recurrence }\end{array}$ & $\begin{array}{l}95 \% \mathrm{Cl} \text { for } \\
\text { odds ratio }\end{array}$ & $P$ \\
\hline \multicolumn{5}{|c|}{ TBR } \\
\hline Age (per year) & & 1.0 & $0.8-1.3$ & 0.8 \\
\hline Sex (male vs. female) & $\begin{array}{c}2 / 66 \\
10 / 169\end{array}$ & 1.6 & $0.3-8.1$ & 0.6 \\
\hline Histotype (papillary vs. follicular) & $\begin{array}{c}10 / 193 \\
2 / 42\end{array}$ & 2.2 & $0.4-11.8$ & 0.34 \\
\hline Extent of thyroidectomy (less than total vs. total ${ }^{\star}$ ) & $\begin{array}{l}11 / 63 \\
1 / 172\end{array}$ & 9.5 & $1.2-78.1$ & 0.04 \\
\hline Radioiodine therapy (no vs. yes) & $\begin{array}{c}11 / 61 \\
1 / 174\end{array}$ & 11.0 & $1.3-94.7$ & 0.03 \\
\hline \multicolumn{5}{|c|}{ NR } \\
\hline Age (per year) & & 0.5 & $0.9-3.4$ & 0.98 \\
\hline Sex (male vs. female) & $\begin{array}{c}5 / 66 \\
18 / 169\end{array}$ & 1.3 & $0.5-3.9$ & 0.6 \\
\hline Lymph node metastases (yes or status unknown vs. no) & $\begin{array}{c}22 / 169 \\
1 / 66\end{array}$ & 2.9 & $0.3-23.6$ & 0.3 \\
\hline Histologic variant of papillary cancer (classical vs. follicular) & $\begin{array}{c}19 / 125 \\
4 / 68\end{array}$ & 1.9 & 1.1-3.4 & 0.02 \\
\hline Extent of thyroidectomy (less than total vs. total ${ }^{\star}$ ) & $\begin{array}{l}12 / 63 \\
11 / 172\end{array}$ & 1.4 & $0.3-1.8$ & 0.5 \\
\hline Lymph node management (incomplete vs. complete ${ }^{\star}$ ) & $\begin{array}{l}16 / 78 \\
7 / 157\end{array}$ & 3.3 & 1.2-8.8 & 0.02 \\
\hline Postoperative radioiodine therapy (no vs. yes) & $\begin{array}{l}15 / 61 \\
8 / 174\end{array}$ & 3.2 & 1.2-7.8 & 0.02 \\
\hline $\begin{array}{l}\text { *See Materials and Methods section for detailed explanations } \\
\mathrm{Cl}=\text { confidence interval. } \\
\text { Odds ratios and confidence intervals (Cls) in bold type denot }\end{array}$ & $\begin{array}{l}\text { these terms. } \\
\text { tatistically significant } v\end{array}$ & & & \\
\hline
\end{tabular}

of primary thyroid surgery also correlated with the likelihood of NRFS ( $P=0.04$; Fig. 7).

In multivariate analysis (Table 4 ), 3 factors were independently statistically significant in predicting NR: Classical papillary histotype increased the risk of this outcome

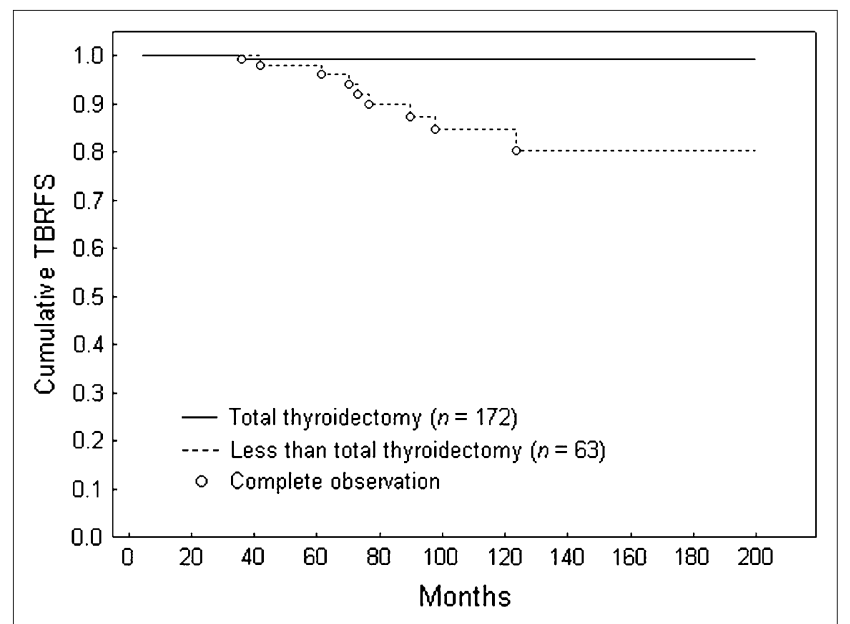

FIGURE 2. TBRFS by thyroid surgery category. Intergroup difference, $P<0.00005$; Cox-Mantel test. Survival curves are cut at $200 \mathrm{mo}$, when only 11 patients were still under observation for this outcome (patients in follow-up: 149 at 5 y, 53 at $10 \mathrm{y}, 5$ at $20 \mathrm{y}$ ). by a factor of $1.9(95 \% \mathrm{CI}, 1.1-3.4 ; P=0.02)$; lack of postoperative radioiodine treatment, by a factor of 3.2 (95\% CI, 1.2-7.8; $P=0.02$ ); and incomplete lymph node management by a factor of $3.3(95 \% \mathrm{CI}, 1.2-8.8 ; P=$ 0.02).

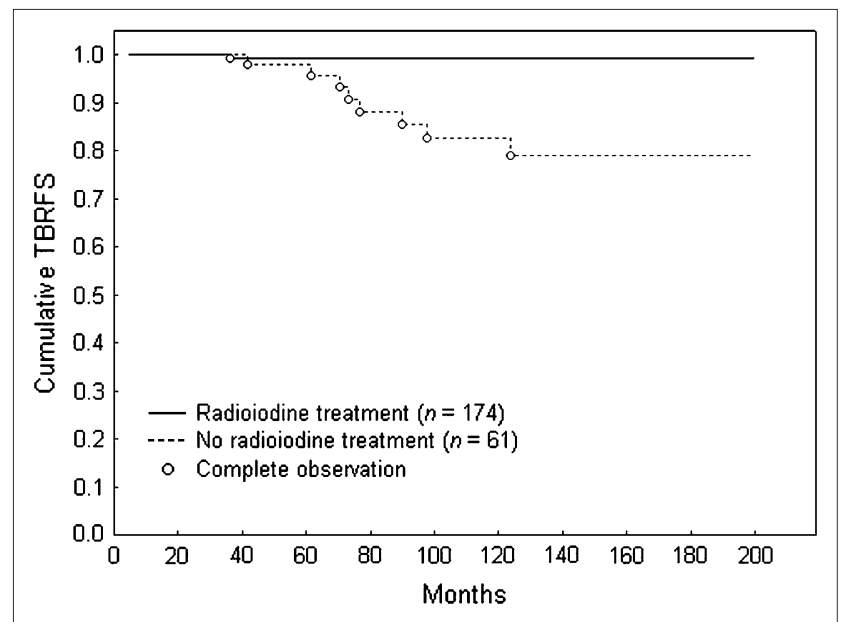

FIGURE 3. TBRFS by radioiodine treatment category. Intergroup difference, $P<0.0005$; Cox-Mantel test. Survival curves are cut at $200 \mathrm{mo}$, when only 11 patients were still under observation for this outcome (patients in follow-up: 149 at $5 \mathrm{y}$, 53 at $10 y, 5$ at $20 y$ ). 


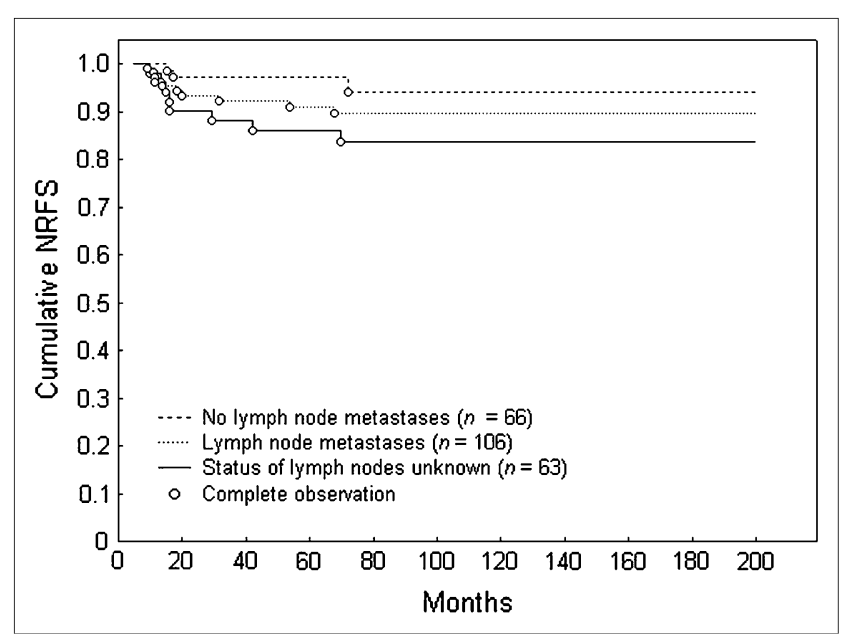

FIGURE 4. NRFS by lymph node metastasis status at end of primary treatment. Intergroup differences: (1) vs. (2), $P=0.04$; (1) vs. (3), $P=0.006$; (2) vs. (3), $P=0.3$; Cox-Mantel test. Survival curves are cut at $200 \mathrm{mo}$, when 15 patients were still under observation for this outcome (patients in follow-up: 141 at $5 \mathrm{y}, 55$ at $10 \mathrm{y}, 8$ at $20 \mathrm{y})$.

\section{DISCUSSION}

The main goal of this study was to assess the contribution of postoperative radioiodine to LRFS in pediatric DTC. To this end, we undertook a multivariate analysis in a large group of children that independently considered the extent not only of radioiodine administration but also of both thyroidectomy and lymph node surgical management, as well as other key patient- and disease-related factors. Only an analysis controlling for the extent of surgery can properly evaluate the effects of radioiodine therapy on LRFS.

Previous multivariate analyses (6,21-23) did not detect recurrence benefits of adjuvant radioiodine therapy in chil-

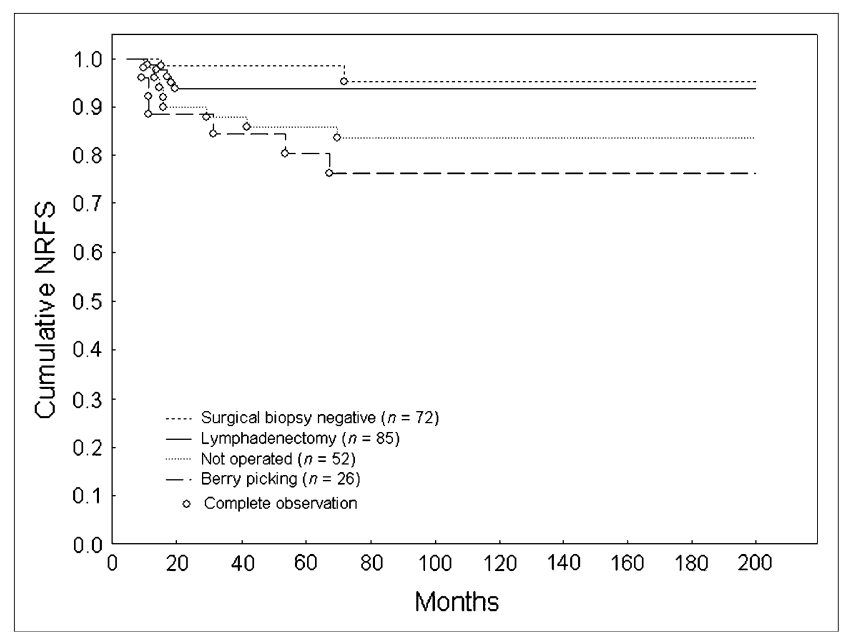

FIGURE 5. NRFS by lymph node surgical management category. Intergroup differences: (1) vs. (2), $P=0.3$; (1) vs. (3), $P=0.014$; (2) vs. (4), $P=0.0006$; (3) vs. (4), $P=0.2$; (1) + (2) vs. (3) $+(4), P=0.02$; Cox-Mantel test. Survival curves are cut at $200 \mathrm{mo}$, when 15 patients were still under observation for this outcome (patients in follow-up: 141 at 5 y, 55 at 10 y, 8 at 20 y).

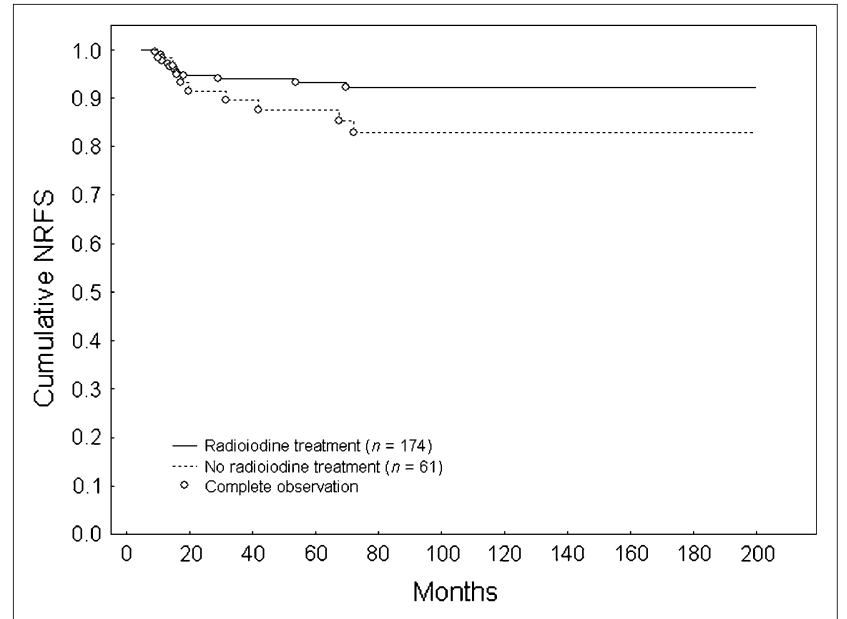

FIGURE 6. NRFS by radioiodine treatment category. Intergroup difference, $P<0.0005$; Cox-Mantel test. Survival curves are cut at 200 mo, when 15 patients were still in observation for this outcome (patients in follow-up: 141 at 5 y, 55 at 10 y, 8 at $20 \mathrm{y})$.

dren, causing many authors to doubt the indication for (23) or to oppose the use of this modality in this setting (17). For example, in a group of patients diagnosed when they were $<21$-y-old and evaluated by Newman et al. (24), progression-free survival did not differ according to the presence or the absence of radioiodine ablation. On the other hand, Chow et al. (15) recently demonstrated with univariate analysis that the LR rate in children was significantly reduced-from $42 \%$ to $6 \%$ - when radioiodine was administered postoperatively. In our previous multivariate analysis (6), the effect of radioiodine on LRFS only trended toward significance $(P=0.07)$. In the present study, with a larger group of children and a longer observation time, our multivariate analysis found a statistically significant benefit from postoperative radioiodine therapy, both in TBRFS and

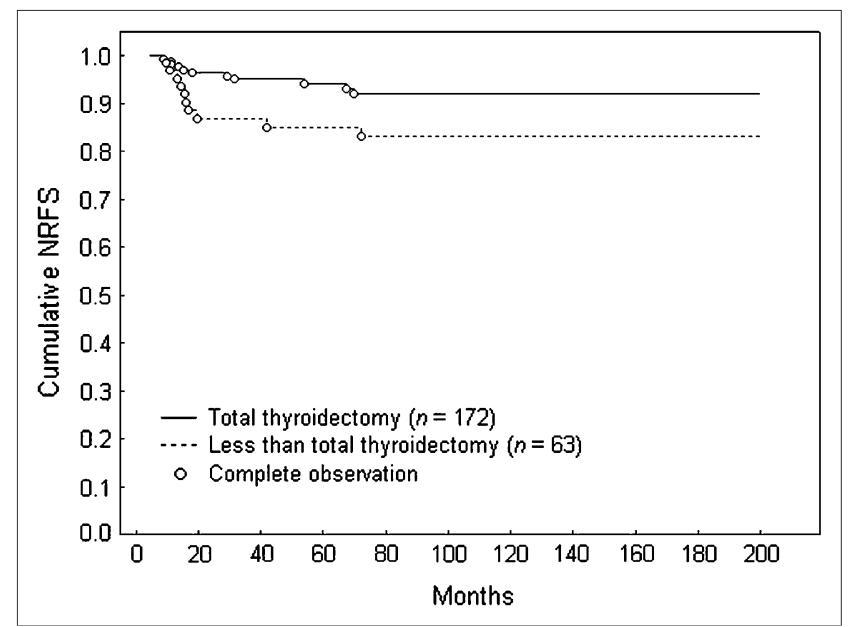

FIGURE 7. NRFS by thyroid surgery category. Intergroup difference, $P<0.05$; Cox-Mantel test. Survival curves are cut at $200 \mathrm{mo}$, when 15 patients were still in observation for this outcome (patients in follow-up: 141 at 5 y, 55 at 10 y, 8 at 20 y). 
NRFS. This evidence supports the routine use of radioiodine after total thyroidectomies, which were used in our group on about $74 \%$ of children. We prefer the term "adjuvant radioiodine" instead of "thyroid remnant ablation," as we demonstrate here that this modality has the therapeutic effect of preventing not only TBR but also NR of DTC, independently of near-total or total thyroidectomy or complete lymph node management. Our finding of the efficacy of radioiodine therapy in preventing LR in children may be explained, at least in part, by the apparently relatively high radiosensitivity of childhood versus adult DTCs, which may in turn be attributable, at least in part, to greater and more frequently detectable sodium-iodine symporter expression in pediatric than in adult DTC cells $(25,26)$.

Fixed radioiodine activities ranging from 1.11 to 3.7 $\mathrm{GBq}$ are typically used for adjuvant ${ }^{131} \mathrm{I}$ therapy. According to Mazzaferri and Massoll (2), use of higher activities (2.775$3.7 \mathrm{GBq}$ ) is more efficient than use of lower activities of $\sim 1.1$ GBq, and we have used the higher range. The higher activities are also more efficient in detecting and treating distant (mainly lung) metastases. As an alternative to this fixedactivity protocol, some centers $(27,28)$ give $3.7 \mathrm{MBq} / \mathrm{kg}$ of body weight (range, 1.85-7.4 MBq/kg). Another alternative, based on Reynolds' calculations, is the use of diagrams adjusting the adult activity to the age of the treated child, with larger decreases in younger children (29). According to this system, a 15-y-old should receive about five-sixth; a 10-yold, one-half; and a 5-y-old, one-third the adult activity.

Our study also demonstrates that near-total or total thyroidectomy and complete lymph node management-that is, modified lymphadenectomy of the lateral neck compartment in all cases of lymph node metastasis, whether diagnosed preoperatively or by intraoperative lymph-node biopsy, or biopsy confirmation of negative node involvementsignificantly and independently lower the LR rate in children with DTC. These results confirm and extend our previous observations (6).

When comparing our LR rate (14\%) with rates in other studies, one must keep in mind that different definitions of recurrence are used in the literature $(10,23,24)$. This difference is especially important when completion surgery is delayed in some patients, as was the case in many of our children, mostly due to slow parental consent. In the present study, LR was diagnosed only when the newly apparent disease was found $>9$ mo after the initial surgery, a criterion that we consider rather strict. Diagnosis of recurrent disease in patients who underwent surgery a few months after primary surgery is, in our experience, nearly always due to an inadequate extent of the first operation-thus, these cases should be interpreted as pseudorelapses. In our study, LR was diagnosed $>12$ mo after initial surgery in $87 \%$ (28/32) of cases-that is, in all but 4 instances ( 3 NRs and 1 TBR). Three of the 4 children with early relapse underwent surgery twice and the time between the second operation and the relapse was $>6$ mo in each case. On the other hand, Welch Dinauer et al. (23) defined time to relapse as the reappearance of cancer $>4$ mo after curative surgery, Borson-Chazot et al. (10) reported only that 7 of 10 NRs occurred during the first postoperative year, whereas Newman et al. (24) studied progression-free, not recurrence-free, survival. We stress that the distinction between progression of residual disease and recurrence after radical surgery may be very difficult in children and, at least in part, contributes to the observed differences in DTC outcome in various reports.

To our knowledge, the present study is the first publication to separately address the issue of TBR and NR in sporadic pediatric DTC. This allows us to avoid the inappropriate conclusion that total thyroidectomy reduces the risk of NR, which would result from our univariate analysis - a finding that probably was biased by more careful lymph node surgery performed in our specialized center during completion thyroidectomy. The rather low number of TBRs in our study can probably be attributed to $>70 \%$ of our patients finishing their initial treatment with total or near-total thyroidectomy followed by radioiodine administration. It should be stressed that in children with less extensive thyroidectomy and no radioiodine after surgery, TBRs occurred in 11 cases, or $17 \%$ of children treated with this approach. One can ask whether the TBR rate in this subgroup justifies the use of more extensive surgical treatment and complementary radioiodine in all patients. Some investigators argue that small residual tumors in thyroid remnants have little impact on the final outcome and that recurrent disease can be easily treated with surgical reintervention $(24,30)$. However, residual malignancy may be more important in children and adolescents than in adults. Because of their higher life expectancy relative to that of adult patients, the time during which children with DTC are at risk for recurrence is much longer, and, in fact, LR rates are much higher in DTC patients diagnosed at $<18-20$ y of age than those of patients diagnosed at 20-45 y of age (2). In our study, the median time to TBR was nearly $7 \mathrm{y}$, and, in 1 patient, it was as long as $20 \mathrm{y}$. Moreover, 4 (44\%) of 9 TBRs were diagnosed after $>10 \mathrm{y}$. These observations suggest that with a longer observation time, one can expect a higher rate of relapses related to less radical primary treatment, especially in the group of patients with one or more factors for increased LR risk.

Another concern over the use of subtotal thyroidectomy in children relates to the risk that, after radioiodine treatment, the radiation dose in large thyroid remnants may be too low to effectively kill cancer cells (31). In this context, it is important to emphasize that we recommend radioiodine therapy to complement-not substitute for-total or near-total thyroidectomy.

Apart from thyroid surgery, another major issue in the treatment of pediatric DTC is lymph node dissection. Lately, more and more authors claim that lymph node metastases adversely influence disease-free survival in patients with DTC $(32,33)$. Indeed, in our univariate analysis, children with lymph node metastases or unknown cervical lymph node status at the primary surgery had a worse prognosis 
than those with lymph node involvement excluded by negative surgical biopsy. Nevertheless, in the multivariate analysis, this effect was overshadowed by the extent of lymph node surgery. If modified lymphadenectomy was performed, the prognosis for NR in children with lymph node metastases at the time of primary treatment did not differ from the prognosis in those with a negative surgical biopsy of the lateral neck lymph node compartment at that time. Conversely, incomplete excision of lymph nodes (berry-picking surgery) or the lack of any pathologic evaluation of lymph node status increased the NR risk by a factor of 3 . These results highlight the substantial role of complete lymph node surgical management in preventing NR. If metastatic lymph node involvement is proven, modified lymphadenectomy is indicated. Also supporting this recommendation is the recent work of Demidchik et al. (21), which showed that lack of neck dissection increased the risk of recurrent nodal disease.

Monitoring the $\mathrm{Tg}$ value is a very helpful tool that not only allows earlier diagnosis of recurrence but also is important for therapeutic decision making in DTC $(18,19)$. Nevertheless, in some cases, its value can be surpassed during follow-up by the earlier detection of NR or TBR using sonography and fine-needle aspiration biopsy (34). In our group of children with recurrent DTC, Tg was usually elevated (but not in all cases) and after successful treatment dropped to values comparable to those of children who never had disease recurrence or distant metastases (Table 3 ). The other issue is the prognostic value of postoperative $\mathrm{Tg}$ values (measured before adjuvant radioiodine therapy), which also is unequivocally proven (35). We did not analyze this aspect in children, because data on postoperative Tg level were lacking in some children who were not referred to our center directly after the surgery and, thus, were not treated by adjuvant radioiodine. The present DTC guidelines refer to $\mathrm{Tg}$ estimation at the time of decision to treat postoperatively with radioiodine in patients with lowrisk DTC, and children fall into this group $(18,19)$. In the recent article of Kim at all (35), the negative predictive value for recurrence in patients having postoperative $\mathrm{Tg}<2$ $\mathrm{ng} / \mathrm{mL}$ was $98 \%$. However, in our childhood population with DTC, 67\% had postoperative serum $\mathrm{Tg}>2 \mathrm{ng} / \mathrm{mL}$ (Table 3), despite the radical operation performed and the overall good outcome. We believe that the question of the predictive cutoff Tg level in children deserves more attention as well as a dedicated study.

We did not extend our analysis of the LR rate into evaluation of distant metastatic recurrence for several reasons. First, in most children, distant metastases are already present at DTC diagnosis, as was the case in at least 40 of 47 , or $85 \%$ of affected patients in our study. Second, for distant metastases, only overall survival-not recurrence-free survival-is an appropriate measurement of the benefit of adjuvant radioiodine treatment in retrospective studies. This is because the presence of distant DTC metastases may be reliably diagnosed only by rxWBS, which, of course, re- quires postsurgical radioiodine administration. Thus, any study comparing the inclusion versus the omission of postoperative radioiodine would be biased by the unequivocal diagnosis of the initial disease stage only in the group receiving this modality. On the other hand, because locoregional disease is accurately diagnosed on the basis of digital examination or US, confirmed by aspiration biopsy, an analysis of the influence of radioiodine treatment on LR was feasible even if some children were not given this intervention. We also decided to omit the tumor status after primary treatment from our analysis, because information was unavailable on multifocality in 82 children $(35 \%)$ and on the capsule status in 142 children $(60 \%)$, who were initially treated outside our center.

In the present study, age did not exert any significant effect on the risk of relapse, in contrast to the findings that have been reported in many other articles $(7,36)$. As already suggested in our previous study (6), our results here demonstrate that an intensive treatment approach in younger children completely eradicates their higher risk of LR.

As noted earlier, our analysis is based on the evaluation of disease-free survival-not overall survival. We strongly believe that reducing LR risk is of utmost importance in young DTC patients, because doing so both reduces cancerrelated mortality (5) and helps avoid the heavy psychologic stress related to the diagnosis and therapy of relapse during formative years. However, because of the long life expectancy of children and adolescents, the rate of treatment complications is of great importance. Indeed, for some authors (30), permanent sequelae of extensive surgery constitute the main premise for avoiding total thyroidectomy. The present study did not include an extensive evaluation of surgical complications, as we are examining this issue in a separate study, based on formal decision analysis, which is similar to that of Kebebew et al. in adult DTC patients (37). Rates of $0 \%-21 \%$ for hypoparathyroidism and $0 \%-17 \%$ for laryngeal nerve palsy are reported in the literature $(8)$. With regard to radioiodine therapy sequelae, we observed a high rate of early minor, transient complications (nausea, vomiting). Concerns about late side effects relate to the possibility of a second malignancy or the impairment of fertility or of pregnancy outcome in young patients treated with radioiodine $(38,39)$. We observed a single case of breast cancer, which we consider more likely to be related to the common genetic background of this malignancy and DTC (40) than to previous radioiodine exposure, as the time between the radioiodine treatment and breast cancer diagnosis was rather short (6 y) and the breast cancer presented at a very advanced stage in this young woman. However, the potential risk of radiation-induced cancer also must be considered when deciding on radioiodine therapy in children (38). No formal analysis of fertility or pregnancy outcome was performed in our group; however, the published data clearly show no deleterious effects of radioiodine therapy on future fertility and offspring (39). 


\section{CONCLUSION}

Our study confirms that children and adolescents with DTC benefit from extensive initial therapy, consisting of total thyroidectomy combined with modified lymphadenectomy performed in cases of lymph node metastases and followed by radioiodine therapy. Such interventions are associated with a substantial decrease of LR risk.

\section{ACKNOWLEDGMENTS}

We acknowledge the contribution of Zbigniew Puch and Aleksandra Krawczyk to the follow-up of our patients and thank Robert J. Marlowe for editorial assistance, provided under an unrestricted educational grant from Genzyme Europe BV (Naarden, The Netherlands). The authors declare that there is no conflict of interest.

\section{REFERENCES}

1. Haugen BR. Initial treatment of differentiated thyroid carcinoma. Rev Endocr Metab Disord. 2000;1:139-145.

2. Mazzaferri EL, Massoll N. Management of papillary and follicular (differentiated) thyroid cancer: new paradigms using recombinant human thyrotropin. Endocr Relat Cancer. 2002;9:227-247.

3. Ringel MD, Levine MA. Current therapy for childhood thyroid cancer: optimal surgery and the legacy of king pyrrhus. Ann Surg Oncol. 2003;10:4-6.

4. Hallwirth U, Flores J, Kaserer K, Niederle B. Differentiated thyroid cancer in children and adolescents: the importance of adequate surgery and review of literature. Eur J Pediatr Surg. 1999;9:359-363.

5. Landau D, Vini L, A'Hern R, Harmer C. Thyroid cancer in children: the Royal Marsden Hospital experience. Eur J Cancer. 2000;36:214-220.

6. Jarzab B, Handkiewicz-Junak D, Wloch J, et al. Multivariate analysis of prognostic factors for differentiated thyroid carcinoma in children. Eur J Nucl Med. 2000;27:833-841.

7. Grigsby PW, Galor A, Michalski JM, Doherty GM. Childhood and adolescent thyroid carcinoma. Cancer. 2002;95:724-729.

8. Haveman JW, van Tol KM, Rouwe CW, Piersdo A, Plukker JT. Surgical experience in children with differentiated thyroid carcinoma. Ann Surg Oncol. 2003;10:15-20.

9. La Quaglia MP, Corbally MT, Heller G, Exelby PR, Brennan MF. Recurrence and morbidity in differentiated thyroid carcinoma in children. Surgery. 1988; 104:1149-1156.

10. Borson-Chazot F, Causeret S, Lifante JC, Augros M, Berger N, Peix JL. Predictive factors for recurrence from a series of 74 children and adolescents with differentiated thyroid cancer. World J Surg. 2004;28:1088-1092.

11. Hay ID, Thompson GB, Grant CS, et al. Papillary thyroid carcinoma managed at the Mayo Clinic during six decades (1940-1999): temporal trends in initial therapy and long-term outcome in 2444 consecutively treated patients. World $\mathrm{J}$ Surg. 2002;26:879-885.

12. Jarzab B, Handkiewicz-Junak D, Wloch J. Juvenile differentiated thyroid carcinoma and the role of radioiodine in its treatment: a qualitative review. Endocr Relat Cancer. 2005;12:773-803.

13. Vassilopoulou-Sellin R, Klein MJ, Smith TH, et al. Pulmonary metastases in children and young adults with differentiated thyroid cancer. Cancer. 1993;71: 1348-1352.

14. Samuel AM, Rajashekharrao B, Shah DH. Pulmonary metastases in children and adolescents with well-differentiated thyroid cancer. J Nucl Med. 1998;39: 1531-1536.

15. Chow SM, Law SC, Mendenhall WM, et al. Differentiated thyroid carcinoma in childhood and adolescence: clinical course and role of radioiodine. Pediatr Blood Cancer. 2004;42:176-183.

16. Sawka AM, Thephamongkhol K, Brouwers M, Thabane L, Browman G, Gerstein HC. Clinical review 170: a systematic review and metaanalysis of the effectiveness of radioactive iodine remnant ablation for well-differentiated thyroid cancer. J Clin Endocrinol Metab. 2004;89:3668-3676.
17. Hay ID, Grant CS, Bergstralh EJ, Thompson GB, van Heerden JA, Goellner JR. Unilateral total lobectomy: Is it sufficient surgical treatment for patients with AMES low-risk papillary thyroid carcinoma? Surgery. 1998;124:958-964.

18. Cooper DS, Doherty GM, Haugen BR, et al. Management guidelines for patients with thyroid nodules and differentiated thyroid cancer. Thyroid. 2006;16:1-34.

19. Pacini F, Schlumberger M, Dralle H, et al. European consensus for the management of patients with differentiated thyroid carcinoma of the follicular epithelium. Eur J Endocrinol. 2006;154:787-803.

20. Kukulska A. Thyroglobulin as a Marker for Differentiated Thyroid Cancer. [MD thesis]. Zabrze, Poland: Medical University of Silesia; 1996.

21. Demidchik YE, Demidchik EP, Reiners C, et al. Comprehensive clinical assessment of 740 cases of surgically treated thyroid cancer in children of Belarus. Ann Surg. 2006;243:525-532.

22. Shapiro NL, Bhattacharyya N. Population-based outcomes for pediatric thyroid carcinoma. Laryngoscope. 2005;115:337-340.

23. Welch Dinauer CA, Tuttle RM, Robie DK, McClellan DR, Francis GL. Extensive surgery improves recurrence-free survival for children and young patients with class I papillary thyroid carcinoma. J Pediatr Surg. 1999;34:1799-1804.

24. Newman KD, Black T, Heller G, et al. Differentiated thyroid cancer: determinants of disease progression in patients $<21$ years of age at diagnosis-a report from the Surgical Discipline Committee of the Children's Cancer Group. Ann Surg. 1998;227:533-541.

25. Patel A, Jhiang S, Dogra S, et al. Differentiated thyroid carcinoma that express sodium-iodide symporter have a lower risk of recurrence for children and adolescents. Pediatr Res. 2002;52:737-744.

26. Faggiano A, Coulot J, Bellon N, et al. Age dependent variation of follicular size and expression of iodine transporters in human thyroid tissue. J Nucl Med. 2004;45:232-237.

27. Schlumberger M, De Vathaire F, Travagli JP, et al. Differentiated thyroid carcinoma in childhood: long term follow-up of 72 patients. J Clin Endocrinol Metab. 1987;65:1088-1094.

28. Hung W, Sarlis NJ. Current controversies in the management of pediatric patients with well-differentiated nonmedullary thyroid cancer: a review. Thyroid. 2002;12:683-702.

29. Reynolds J. Comparison of I-131 absorbed radiation doses in children and adults: a tool for estimating therapeutic I-131 doses in children. In: Robbins J, ed. Treatment of Thyroid Cancer in Children. Washington, D.C.: U.S. Department of Energy and U.S. Department of Commerce: Technology, Administration, National Technical Information; 1993:127-135.

30. van Santen HM, Aronson DC, Vulsma T, et al. Frequent adverse events after treatment for childhood-onset differentiated thyroid carcinoma: a single institute experience. Eur J Cancer. 2004;40:1743-1751

31. Maxon HR. Quantitative radioiodine therapy in the treatment of differentiated thyroid cancer. Q J Nucl Med. 1999;43:313-323.

32. Salvesen H, Njolstad LA, Akslen LA, Albrektsen O, Soreide O, Varhaug JE. Papillary thyroid carcinoma: a multivariate analysis of prognostic factors including an evaluation of the p-TNM staging system. Eur J Surg. 1992;158:583-589.

33. Robie D, Welch Dinauer CA, Tuttle RM. at al. The impact of initial surgical management on outcome in young patients with differentiated thyroid cancer. J Pediatr Surg. 1998;33:1134-1140.

34. Torlontano M, Crocetti U, Augello G, et al. Comparative evaluation of recombinant human thyrotropin-stimulated thyroglobulin levels, ${ }^{131} \mathrm{I}$ whole-body scintigraphy, and neck ultrasonography in the follow-up of patients with papillary thyroid microcarcinoma who have not undergone radioiodine therapy. J Clin Endocrinol Metab. 2006;91:60-63.

35. Kim TY, Kim WB, Kim ES, et al. Serum thyroglobulin levels at the time of ${ }^{131} \mathrm{I}$ remnant ablation just after thyroidectomy are useful for early prediction of clinical recurrence in low-risk patients with differentiated thyroid carcinoma. J Clin Endocrinol Metab. 2005;90:1440-1445.

36. Alessandri AJ, Goddard KJ, Blair GK, Fryer CJ, Schultz KR. Age is the major determinant of recurrence in pediatric differentiated thyroid carcinoma. Med Pediatr Oncol. 2000;35:41-46.

37. Kebebew E, Duh QY, Clark OH. Total thyroidectomy or thyroid lobectomy in patients with low-risk differentiated thyroid cancer: surgical decision analysis of a controversy using a mathematical model. World J Surg. 2000;24:1295-1302.

38. Rubino C, de Vathaire F, Dottorini ME, et al. Second primary malignancies in thyroid cancer patients. Br J Cancer. 2003;89:1638-1644.

39. Schlumberger M, De Vathaire F, Ceccarelli C, et al. Exposure to radioactive iodine-131 for scintigraphy or therapy does not preclude pregnancy in thyroid cancer patients. J Nucl Med. 1996;37:606-612

40. Cybulski C, Gorski B, Huzarski T, et al. CHEK2 is a multiorgan cancer susceptibility gene. Am J Hum Genet. 2004;75:1131-1135. 